\title{
The advance treatment on neonatal respiratory distress syndrome
}

\author{
Volume 6 Issue 5 - 2017 \\ Yan Wang \\ The American Physiological Society, USA \\ Correspondence: Yan Wang, MD, PhD., The membership \\ of the American Physiological Society, Contact Address: Rm \\ I I OI, Cell 26/99 JinHe Rd.Shanghai, CN, Tel I3482646536, Fax \\ 862000000000, Email wanggy@mail.com
}

Received: April 27, 2017| Published: May 09, 2017 surfactant deficiency disorder (SDD) or called hyaline membrane disease (HMD), it is occurred in premature neonatal often born before 37 weeks in gestation; the incidence and severity are related with the gestational age from about 50\% incidence rate at 26-28weeks and about $25 \%$ at $30-31$ weeks in new born. In etiology, the syndrome is caused by a lack of surfactant in the lung, and the substance has function to keep lung fill with air and maintain alveolar sac from deflated, also by research, genetic abnormal including gene of cystic fibrosis conductance regulator or Nitric Oxide enzyme to lung development is relationship with the NRDS, ${ }^{1,2}$ meanwhile, intrauterine infection, premature birth, diabetes's mother or rapid labor can increase the risk to NRDS occurring.

\section{Clinical observation and measurement}

According to clinical observation, NRDS can appear in shorter time within minutes of birth, the manifestation usual present as skin discoloration, nare flaring, grunting, apnea, abnormal breathing pattern, motivation unresponsive or decreased urine output. Under the disease progresses, the neonatal may develop prolonged cessations of breathing, respiratory failure and multple organic failure. ${ }^{1}$ In lab measurement, blood gas analysis shows low xyogen concentration and excess acid producing in the body fluid; chest X-ray presents typically a ground glass appearance to the lung; in others measurements, there may explore that Metabolic disorder, low blood pressure, patent ductus arteriosus, brain bleeding, pulmonary herpentension or more additional defects.

\section{Treatment and medication}

In general, current clinical management to NRDS, life support therapy is in the first aid, which includes oxygen supply, life signs monition, fluid and nutrition supply and respiratory assistantcontinuous positive airway pressure (CPAP) is the simple device to send air through nose to help keep the airways open. In medicine treatment, extra surfactant extracted from organic solvent and Glucocorticoid medicine at smaller volume have suggested to use earlier in the aim to improve the clinical condition of the new born with NRDS..$^{1,3,4}$

\section{In advancement treatment}

Reviewed on NRDS treatment, more achievement has arrived from Pediatrics, Physiology and genetic study, since it provides more insights into the observation about NRDS, these induce NRDS mortibility rate of NRDS to low than 50\% from higher about 70-90\% in the history, thus, the body weight for the treated new born can be less than $1500 \mathrm{~g}$.

\section{The advancement study including}

In surfactant replacement therapy, Knowledge to it's molecular constructure and biochemistry activity study, surfactant can separate into four protein of SP-A,B, C and D, and the replacement treatment has used two SP-B and SP-C from them to treat NRDS, this choice for surfactant replacement is in the goal to get more effective clinical result., ${ }^{2,3}$ For glucocorticoid therapy, it reported glucocorticoid gene related with lung development and it presents that woman received betamethasone can accelerate fetal lung maturity, at later time, glucocorticoid to neonatal direct therapy fret delivery also can see primary outcome to enhance pulmonary maturity. ${ }^{4,5}$ Breathing management by ventilator machine has new types to match various situation required to NRDS as high frequency ventilation, low volume ventilation, assist control ventilation and neutrally adjusted ventilator assist. ${ }^{6}$ For extracorporeal membrane oxygenation (ECMO), it is the potential treatment to provide oxygenation through an apparatus that imitates the gas exchange process of the lungs.

After above, the considering to defend infection and seqealae syndrome as bronchopulmonary dysplasia (BPD), which is also at the advancement treatment to keep the new born's life quality for the future.

\section{Acknowledgments}

None.

\section{Conflicts of interest}

Author declares there are no conflicts of interest.

\section{Funding}

None. 


\section{References}

1. Wang Y, Zhnag LN, Shen WT . The influence of blood serum nitric oxide and endothlin in infant pneumonia respiratory failure. Journal of Applied Clinical Pediatrics. 1998;13:S34-S35,

2. Rubarth LB, Quinn J . Respiratory development and respiratory distress syndrome. Neonatal Network. 2015;34(4):231-238.

3. Whitsett JA. The molecular era of surfactant biology. Neonatology. 2014;105(4):337-343.
4. Haas DM, Lai D, Sharma S, et al. Steroid pathway genes and neonatal respiratory distress after betamethasone use in anticipated preterm birth, Reproductive Sciences. 2016;23(5):680-686.

5. Nelson R. Steroid reduce respiratory problems in late preterm infants Lancet Respir Med. 2016;4(3):180.

6. Shetty S, Hunt K, Peacock L. Crossover study of assist control ventilation and neurally adjusted ventilatory assist. Eur J Pediatri . 2017;176(4):509-513. 